\title{
Dynamic Finger Movement Tracking and Voice Commands Based Smart Wheelchair
}

\author{
Fahad Wallam and Muhammad Asif
}

\begin{abstract}
-many disable persons are able to operate a joystick to control the electric wheelchair but on the other hand, many people do not have the legerdemain necessary to operate a joystick. Moreover, many of them face difficulty to avoid obstacles. The aim of this paper is to implement a multi-control system to control the movement of the wheelchair by integrating finger movement tracking system, a small vocabulary speaker dependent based word recognition system and a group of monitoring sensors in order to avoid obstacles. Moreover, a joystick control system is also implemented in order to facilitate the patients that are able to use the joystick. The theme is accomplished by using a micro-controller with digital signal processor for isolated word recognition and sets of sensors. The results are also given at the end of the paper.
\end{abstract}

Index Terms - Finger movement tracking, speech recognition, gesture recognition, fingerprints.

\section{INTRODUCTION}

Despite rapid technological and scientific progress in devices for persons with disability, there has been very little advancement in the wheelchair design over the last 200 years. The folding wheelchair came in 1933, and powered wheelchairs were developed in the early 1970s [1]. New materials such as plastics, fiber-reinforced composites and beryllium-aluminum alloys have found their way into the design and manufacturing of lighter, stronger and more reliable wheelchairs [2].

Various electric wheelchairs with computer-controlled mechanism have been developed in the recent years such as "WALKY", "Tin Man", "CALL smart chair", and "Nav-Chair" [3-6]. These electric wheelchairs may use a broad variety of sensors such as ultrasonic and infrared sensors, cameras, encoders, accelerometers and gyroscopes, with several desired control devices such as joystick, pressure pads, etc in order to provide ease and comfort [7]. Electric wheelchair systems integrated with customized user interfaces, various sensors, and different types of controllers, can make the function and controlling of a wheelchair much simpler and safer and make it more stable and handy for disabled persons [8]. Appropriate algorithms for controlling

Manuscript received January 9, 2011; revised July 11, 2011.

Fahad Wallam is with Electronic Engineering Department, Sir Syed University of Engineering and Technology, Karachi (e-mail: fwallam@gmail.com).

Muhammad Asif is with Electronic Engineering Department, Sir Syed University of Engineering and Technology, Karachi (e-mail: muasif@ssuet.edu.pk). the wheelchair aid the user in avoiding obstacles such as walls, moving obstacles like people and other wheelchairs, planning collision-free paths and moving safely in jumbled environments without taking much of the user input [9-12].

In the joystick based electric wheelchairs, the joystick serving as the main control interface for the wheelchairs, has the advantage of detailed movement direction and speed control, it has the disadvantages of requiring complex wrist movement that becomes difficult for certain disabled persons and aged ones and may result in inadequate and improper control leading to accidents $[13,14]$. On the other hand there are persons with disability that can operate the joystick very conveniently and safely. It is therefore useful to consider a control system for the electric wheelchair that contains both the joystick based control as well as non-joystick based control systems that do not need the complex wrist movement, in order to fulfill the disabled persons need.

This paper proposes a new approach to the same problem of a better control, as discussed above, for the wheelchair by integrating both the joystick and non-joystick based controls into the wheelchair along with the group of sensors and actuators with gears. The non-joystick based control systems include the finger movement tracking control system and voice command control system. These three controls help almost all categories of disabled persons to derive the wheelchair easy and safe.

In the history of technology, few inventions such as micro-controllers and digital signal processors have changed the world revolutionary. The exponential increase in the computer power while decreasing cost and size of the micro-controllers and processors have made our life not easy but fill full of charm in it. Because of their advantages, the TMS320C6711 digital signal processor (DSP) and AVR micro-controller are used in this work. The DSP is used for implementing speaker dependent speech recognition system for capturing vocal commands for the wheelchair and the micro-controller is used to track the finger movement signals and captures the joystick signals as well. The micro-controller also serves as the main interface among the three control systems and the sensors and actuators.

The rest of the paper is organized as follows: Section II outlines a general idea of the overall system operation. The detailed discussion of the system is giving in Section III. In this section, the three control systems, finger movement tracking, voice command and joystick controls, are explained in very detail. Furthermore, a discussion about monitoring sensors is also presented. Finally, the results and conclusion are presented in Section IV and V respectively 


\section{SYSTEM OVERVIEW}

In this work, micro-controller, digital signal processor, controlling circuitry and sensors are implemented on a simple wheelchair along with actuators. The main theme is to control the wheelchair according to the signals from the input source. The input source can be the voice, the finger movement signals or the joystick. This is illustrated in figure 1 . The switches are provided to select the appropriate input signal source.

The TMS320C6711 DSP kit is used for speech recognition task. It processes the speech signals and generates appropriate analog signals to command the micro-controller so as to control the motion of the wheelchair according to the word spoken. Four words, Left, Right, Up and Stop, are used as voice commands

Special sensors are used to track the movement of the fingers. These sensors generate analog signals, which are translated by the micro-controller to control the wheelchair. The ultrasonic sensors are used to detect the obstacles while optical encoders enable the system to control the speed of the chair conveniently. The micro-controller's final decision is based on the signals received from the monitoring sensors so as not to put the user in danger.

\section{SYSTEM CONTROL}

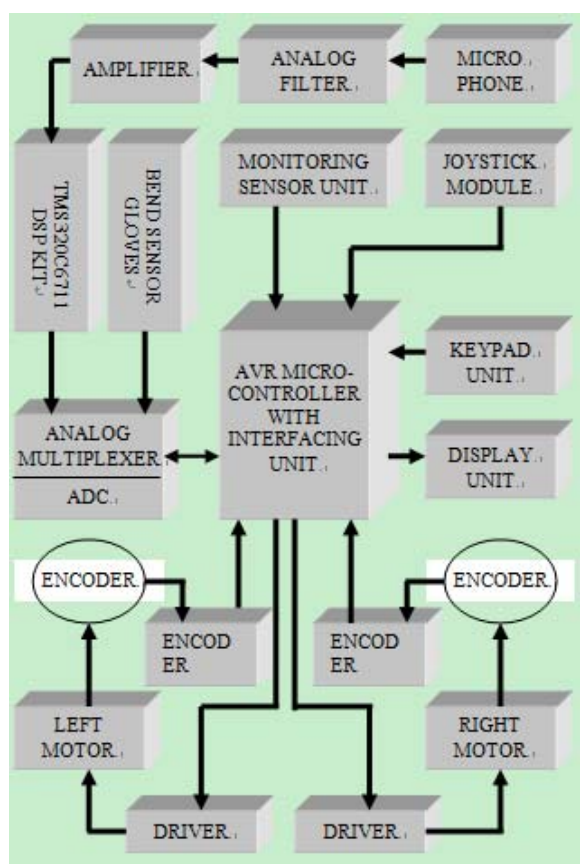

Figure 1: Block Diagram of the System

The wheelchair can be driven either by selecting the finger movement tracking control, the voice command control or the joystick control. There are three switches given to select one control system at any time for driven the wheelchair. When a particular control switch is selected, it gives interrupt to the micro-controller and the micro-controller adjusts itself for the control-system selected.

\section{A. FINGER MOVEMENT TRACKING CONTROL SYSTEM:}

In this work, movement tracking is chosen because of its superiority over gesture recognition, as it has no boundaries in order to capture any motion rather than to wait for a special sign being made in gesture recognition. In gesture recognition, gestures are usually preprogrammed in the application (i.e., a finite set of very specific body positions) and the user is trained to use these predefined gestures to control the system. Also, computer recognition of hand gestures is difficult in complex scenes [15].

The gestures are static, discrete, and restrictive. As a difference, movement tracking involves constant monitoring and dynamic and continuous tracking of the user who uses a series of movements to continue with the application. In movement tracking, the application is to be trained instead of the user. It emphasizes the natural interaction between the user and the application. To achieve the natural interaction, the application must learn to adapt the user's movement rather than the user trying to satisfy the requirements and limitations of the application.

In this system, the wheelchair can be controlled by a combination of three fingers. One finger controls the speed of the wheelchair while the other two fingers control the directions of the wheelchair. There is flexibility that we can use only two fingers instead of three to control the direction while letting the system to control the speed automatically. For the purpose of tracking the movement of fingers, the flex-sensors are used. The sensors are fastened at the finger position on the gloves as shown in figure 2. The resistive bend (flex) sensors are the sensors that change their resistance when they are bent. A flat flex-sensor has a resistance of about $10,000 \mathrm{Ohms}$ (that is, at 0 degree, its resistance is 10,000 Ohms.) As the sensor is bent, its resistance increases to about $35,000 \mathrm{Ohms}$ at 90 degrees as shown in figure 3 [16].

The flex-sensors provide the bending angle information of the fingers to the system. The system compares this information with those obtained during training and finds a command to send to the actuators to control the wheelchair. After verifying that there is no conflict between the command it ready to send to the chair and the data obtained from the monitoring sensors, it sends the command to the actuator so as the chair to act accordingly. The block diagram for the system is presented in figure 4 .

\section{B. VOICE COMMAND CONTROL SYSTEM:}

Speech recognition has been actively studied since $1960 \mathrm{~s}$, however recent developments in computer and digital signal processing technology have improved speech recognition capabilities. Although, now days, speech recognition is a very complex problem. It involves many techniques and algorithms, which require high computational requirements [17].

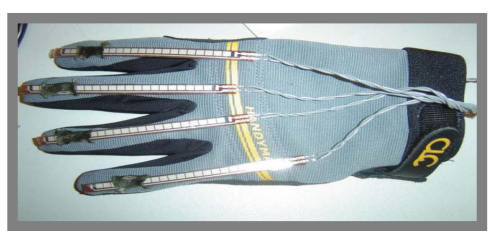

Figure 2: Gloves fastened with Resistive Bend Sensors.

After the introduction of specialized digital signal processors, the real-time digital signal processing is more easily implemented than before [18]. However, speech recognition has a very important role in many application 
fields [19-22]. Various studies in the last few years show good results in both research and commercial applications than as before [23-25].
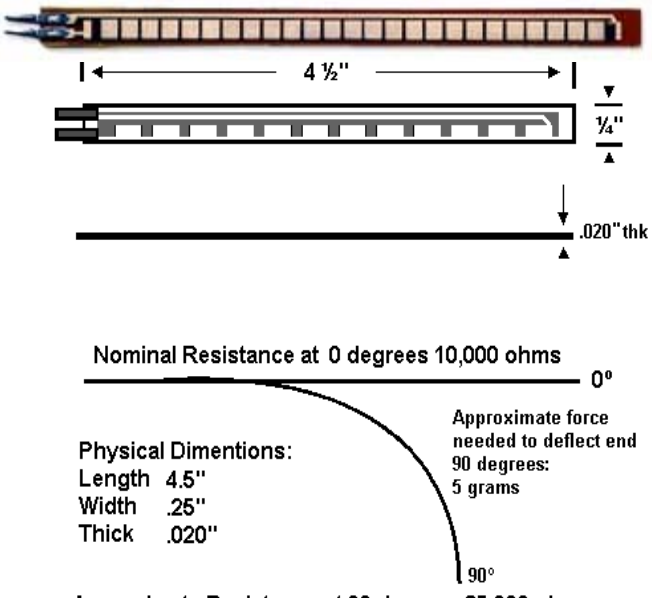

Approximate Resistance at 90 degrees 35,000 ohms

Figure 3: Flex Sensor and its Characteristics

The model of the speech recognition used in the voice command control system, implemented on TMS320C6711 DSP kit, is shown in figure 5. The signals from the microphone are first passed from the analog low pass filter of the second order with a cut off frequency of $3 \mathrm{KHz}$ and amplifier and then digitizes through analog to digital converter integrated in the kit. The digitized signals are then passed through the word detection algorithm. Here, the processor calculates the average energy of the noise in the environment during training and uses it as a threshold point for detecting the start of the word.

$$
E=\frac{1}{3000} \sum_{i=0}^{2999}\left|x_{i}\right| \mathrm{z}
$$

When the energy of the incoming samples exceed from this threshold value, the system thinks as the word is being spoken and captures only the first 3000 incoming samples. The captured signals are then passed through normalization algorithm because sometimes we speak loudly and sometimes we speak gently. The energy of the word spoken loudly is much more greater than that of spoken gently. So, there is a need for normalization. One method of normalization that is used in this work is to determine the maximum value in the samples and then divide each sample with this value. After normalization, the samples are multiplied with a certain gain value.

The signals are then passed through five digital FIR band pass filters whose band pass frequencies are shown in the block diagram. The Blackman window is used for the filter and the function [26] is given in figure 4.

The samples from each Band Pass filter are grouped into frames. The length of each frame is 200 (that is, there are 200 samples in each frame). The total number of samples is 3000 therefore the number of frames for each filter is 15 $(3000 / 200=15)$.

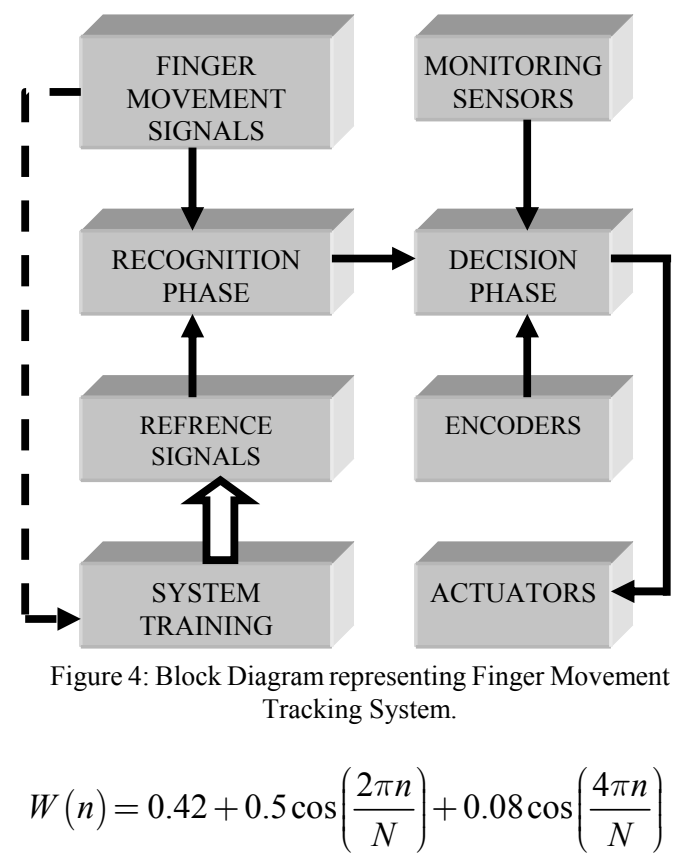

The fingerprint generation is the most important step in the speech recognition task. In this step, the samples are squared and accumulated together in each frame from each filter. It is more easily understood by having look at the following equation:

$$
F_{K}(N)=\sum_{l=0}^{199} x_{K, N}^{2}(l)
$$

where, $N$ represents the number of frames and $N=1,2,3 \ldots 15$ as there are 15 frames from each filter; $K$ represents the band pass filters and $K=1,2 \ldots 6$ as there are 6 band pass filter. Likewise, the index $l=0$ to 199 represents the samples in each frame, as there are 200 samples in each frame. In this way, there are 15 fingerprints (one for each frame) from each filter and as there are 6 filters so the total number of fingerprints are $90\left(15^{*} 6=90\right)$ for each spoken word.

In the fingerprints processing step, the fingerprints of the spoken word are compared with fingerprints of the all the words in the dictionary that are obtained during training. Two techniques are combined for achieving the recognition task. First, the system calculates the corresponding difference between the fingerprints of the spoken word and the fingerprints of each word in the dictionary as:

$$
\operatorname{Diff}_{K, M}(L)=\left|F d_{K, M}(L)-F s_{K}(L)\right|
$$

where, $L$ represents the number of fingerprints from each filter and $L=1,2,3 \ldots 15 ; \mathrm{K}$ represents the band pass filter and $K=1,2 \ldots 6 ; F d$ represents the fingerprints of the $M^{\text {th }}$ word in the dictionary where $M=1,2 \ldots 4$ as in this work four words (Left, Right, Up and Stop) are stored in the dictionary. Also, Fs represents the fingerprints of the spoken word. As there are four words so the result is four sets of elements with each set consist of 90 elements. Then, the values of all four sets are compared correspondingly with one another and the value with the smallest magnitude is marked. Finally, the marked values are counted and the set with the largest value is selected. 


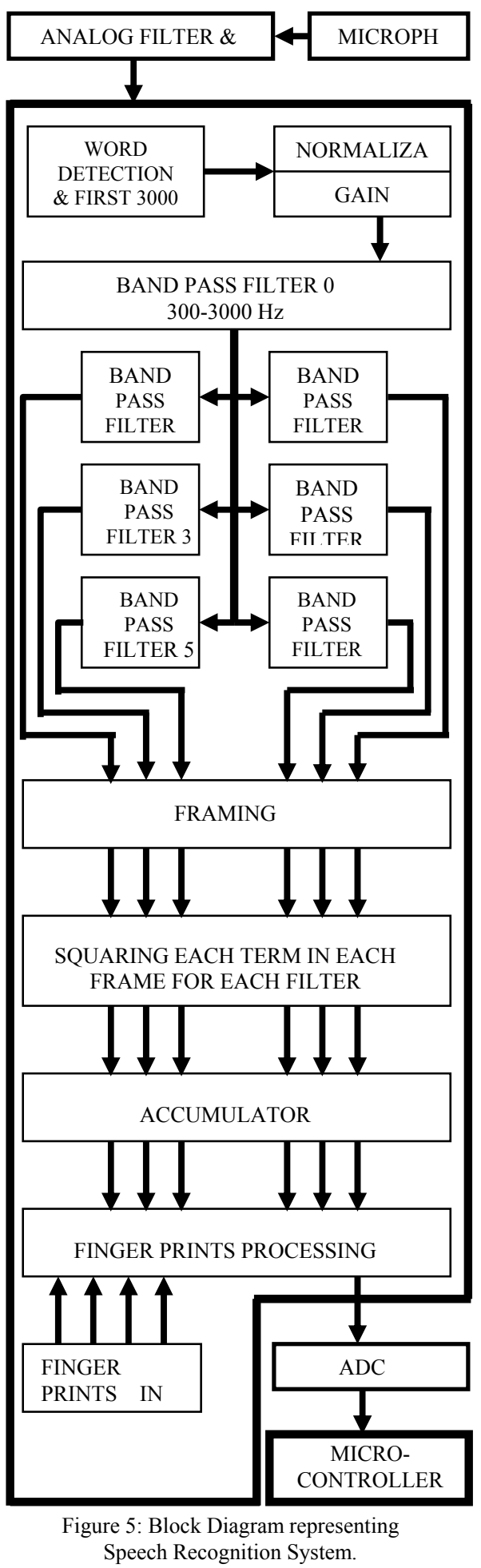

The second technique used in this work is to calculate the correlation between the spoken word and all the four words in the dictionary. As a result, four values are generated. All these four values are compared with one another and the value with the largest magnitude is selected. The coefficient of correlation [27] is given as:

$$
\mu=\left|\frac{n\left(\sum x y\right)-\left(\sum x\right)\left(\sum y\right)}{\left\{\left[n\left(\sum x^{2}\right)-\left(\sum x\right)^{2}\right] *\left[n\left(\sum y^{2}\right)-\left(\sum y\right)^{2}\right]\right\}^{\frac{1}{2}}}\right|
$$

where, $\mathrm{x}$ represents the fingerprints of the dictionary word and $y$ represents the fingerprints of the spoken word.

If the two results point to the same dictionary word, the system thinks as it recognizes the word otherwise, the system discards the spoken word and wait for the next voice command.

\section{JOYSTICK CONTROL SYSTEM:}

The digital joystick is interfaced to the micro-controller by using the interfacing circuitry. The interfacing circuitry consists of IC "74C922" along with the supporting components. The IC is interfaced to the micro-controller in the hardware interrupt mode. In this mode, whenever there is any activity on the joystick, the IC sends an interrupt signal to the micro-controller and also gives the binary input for that particular movement to the micro-controller if the joystick control mode has been selected as the control system for the wheelchair.

\section{MONITORING SENSORS:}

Sets of ultrasonic sensors (transmitter and receiver) are placed in the front as well as at the left and right sides of the wheelchair in order to avoid obstacles and to maintain a safe distance from the obstacles.

Ultrasonic sensors are chosen as monitoring sensors because it is not light sensitive as infrared sensors are. The other property of ultrasonic sensors is to produce mechanical vibrations so as to reflect ultrasonic waves from transparent obstacles such as glass. For moving obstacles like people, with the help of these ultrasonic sensors, the system measures the distance of the wheelchair from the obstacles and by continuously measuring the distance, the system reduces the speed of the chair until the obstacle disappears from its path or stops the chair if a safe distance is reached. The system calculates the distance of the wheelchair from the obstacle by using the following formula:

$$
D=\frac{v^{*} t}{2}
$$

where, $D$ is the distance of the wheelchair from the obstacle, $v$ is the velocity of sound $(340 \mathrm{~m} / \mathrm{s})$ and $t$ is the time from sending an ultrasonic pulse to receiving the very pulse.

\section{RESUlTS \& Discussions}

The system is interfaced and checked with the Personal Computer (PC). The Matlab is used for the simulation on computer. The microphone along with the analog filter and amplifier is connected with the PC. Also, the finger movement tracking system (that includes sensory gloves, micro-controller and ADC) is also interfaced with the PC using serial interface.

The table 1 represents the angles obtained during the training of finger movement tracking system. In this test, two fingers and a thumb are used to control the wheelchair. The thumb controls the speed while the other two fingers control the direction of the wheelchair. This table shows that the wheelchair operates if finger position appears between these angles and if any of the two fingers appears in the angle range set for the other during training, the system commands to stop the wheelchair and consider it as a bad command.

In the voice command control system, the samples of the words after passed through accumulator (fingerprints generation) are stored in the memory during training and called them as dictionary words. The sample words include 
left, right, up and stop. The sample length is selected as 3000 which are captured at a sampling rate of 8000 samples per second that is the voice is recorded for 0.375 seconds $(3000 / 8000=0.375)$ which is the enough time to say a complete word.

TABLE 1: TESTING RESUltS

\begin{tabular}{|c|c|c|c|c|}
\hline & SPEED & LEFT & RIGHT & STOP \\
\hline $\begin{array}{l}\text { Thumb's } \\
\text { Angle }\end{array}$ & $0^{\circ}-90^{\circ}$ & - & - & \multirow{3}{*}{$\begin{array}{c}\text { The } \\
\text { Wheelchair } \\
\text { will be } \\
\text { stopped } \\
\text { other wise. }\end{array}$} \\
\hline $\begin{array}{c}1^{\text {st }} \\
\text { Finger's } \\
\text { Angle }\end{array}$ & & $30^{\circ}-55^{\circ}$ & $\begin{array}{c}\text { Angle } \\
\text { other than } \\
35^{\circ}-60^{\circ}\end{array}$ & \\
\hline $\begin{array}{l}2^{\text {nd }} \\
\text { Finger's } \\
\text { Angle }\end{array}$ & - & $\begin{array}{c}\text { Angle } \\
\text { other than } \\
30^{\circ}-55^{\circ}\end{array}$ & $35^{\circ}-60^{\circ}$ & \\
\hline
\end{tabular}

The results obtained show a prototype that fulfills a large degree of the requirements of people who need a wheelchair in order to be able to move around. It may only require the help of a person to configure the system initially.

\section{CONCLUSION \& FUTURE WORKS}

Recent improvements in the technology are making lives easier for everybody. This work is to help the disabled persons by implementing joystick, finger movement tracking and voice command based control system for the wheelchair.

This paper addresses a hardware design of a multi-control system, implemented using AVR micro-controller act as the main controller and TMS320C6711 DSP kit, which has a large on board memory to store sound parameters, is used for speech recognition task. A very simple real time algorithm has been developed for the speech recognition task and bends resistive sensors, which are used for the finger movement tracking, interfaced to the micro-controller. Finally, the wheelchair understands the signals coming from the control system and reacts accordingly. The efficiency of the voice command control system can be further improved by implementing neural network based algorithms.

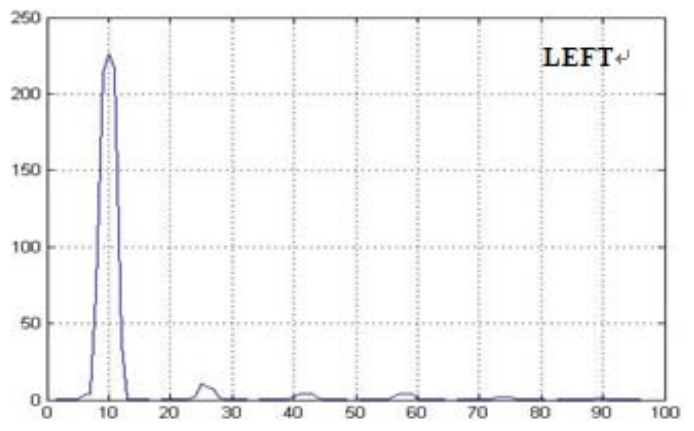

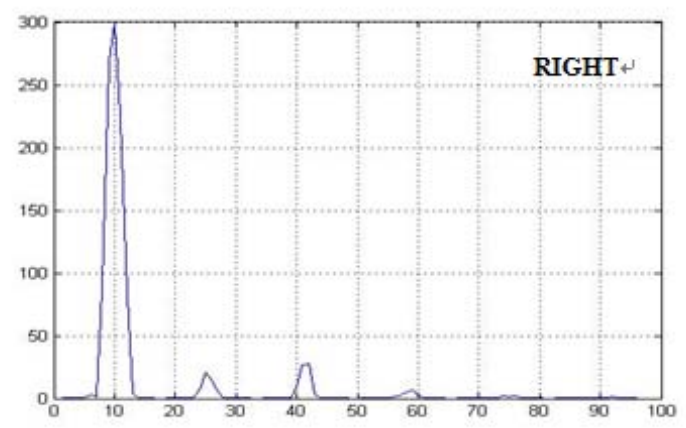
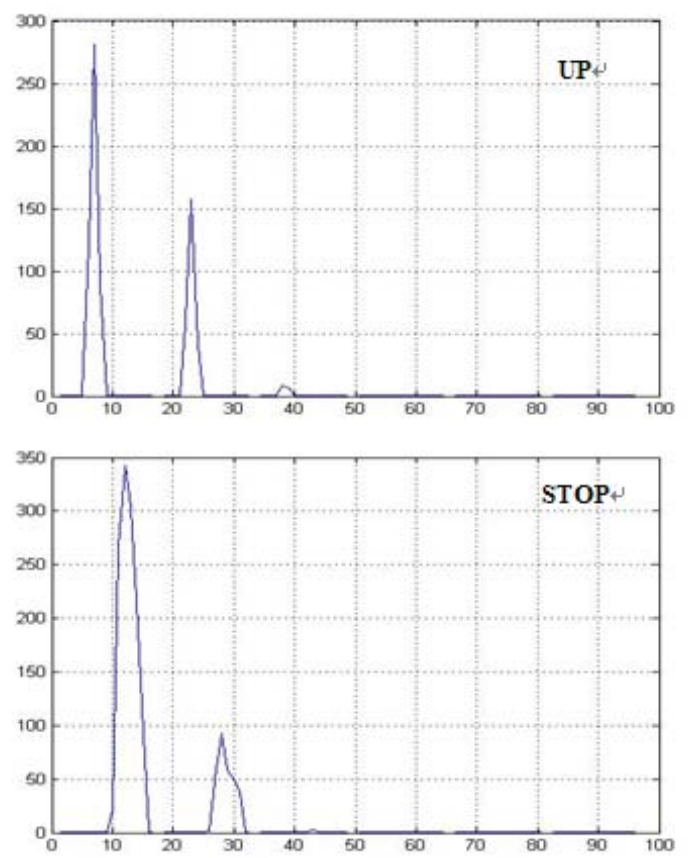

Figure 6: Fingerprints of four different words

\section{ACKNOWLEDGEMENT}

Authors would like to thank Dr. Muhammad Naim Wallam for his kind support without which this work would not be possible. Special thanks to Ms. Samreen Amir and Mr. Fawad Shaukat, Assistant professors at Electronic Engineering Department, Sir Syed University of Engineering and Technology, for their guidance and support for writing and organizing this work.

\section{REFRENCES}

[1] Teitelman, "De-handicapping the handicapped. Forbes", September 24, 1984.

[2] C.A. McLaurin and P. Axelson, "Wheelchair standards: an overview", Journal of Rehabilitation Research and Development (Clinical Supplement), 1990, 27(2): pp. 100-103.

[3] J.D. Nisbet, I.R. Loudon and J.P. Odor, "The CALL Center smart wheelchair". In Proceedings of $1^{\text {st }}$ International Workshop on Robotic Applications to Medical and Health Care, 1988, Ottawa, Canada.

[4] D.A. Bell, J. Borenstein, S. Levine, Y. Koren and L.A. Jaros, "An assistive navigation system for wheelchairs based upon mobile robot obstacle avoidance". In Proceedings of the IEEE International Conference on Robotics and Automation, May 8-13, 1994, San Diego, CA, pp. 2012-2017.

[5] D. Miller and M. Slack, "Design and testing of a low-cost robotic wheelchair prototype", Autonomous Robots, 1995, 2(1): pp. 77-88.

[6] Håkan Neveryd and Gunnar Bolmsjö, "WALKY, A mobile robot system for the disabled". In Proceedings of the $4^{\text {th }}$ International Conference on Rehabilitation Robotics (ICORR), June, 1994, Wilmington, Delaware, USA, pp. 137-141. 
[7] M3S: A general-purpose multiple-master multiple-slave intelligent interface for the rehabilitation environment, International Standards Organization, 1995.

[8] J. M.Ford and S. J. Sheredos, "Ultrasonic head controller for powered wheelchairs". Journal Of Rehabilitation Research and Development, 1995, 32(3): pp. 280-284.

[9] P.F. Muir and C.P., "Neuman, Kinematic modeling for feedback control of an omni-directional wheeled mobile robot", In I.J. Cox and G.T. Wilfong (eds.) Autonomous Robot Vehicles, Springer Verlag, 1990, pp. 25-31.

[10] D.A. Bell, S.P. Levine, Y. Koren, L.A. Jaros and J. Borenstein, “An identification technique for adaptive shared control in human-machine systems". In Proceedings of the 15th Annual International Conference of the IEEE Engineering in Medicine and Biology Society, October 1993, San Diego, CA, pp. 1299-1300.

[11] J. Borenstein and Y. Koren, "Tele-autonomous guidance for mobile robots", IEEE Transactions on Systems, Man and Cybernetics, 1991, 17(4), pp. 535-539.

[12] U. Borgolte, R. Hoelper, H. Hoyer, H. Heck, W. Humann, J. Nezda, I. Craig, R. Valleggi and A. M. Sabatini, "Intelligent control of a semi-autonomous omni-directional wheelchair". In Proceedings of the 3rd International Symposium on Intelligent Robotic Systems '95 (SIRS 95), July 10-14, 1995, Pisa, Italy, pp. 113-120.

[13] J. Kono and J. Inada, "Special Characteristics of Tooth Brushing Movement in Elementary School and the Elderly". The Japanese Society for Dental Health, Vol.58, No.3, pp 91-92.

[14] S. Yokota, H. Hashimoto, Y. Ohyama and J. She, "Electric wheelchair controlled by human body motion", Journal of Robotics and Mechatronics, 2010, Vol. 22, No. 4, pp 439-446.

[15] V.I. Pavlovic, R. Sharma, and T.S. Huang, "Visual interpretation of hand gestures for human-computer interaction: A review", IEEE Transactions on Pattern Analysis and Machine Intelligence, July 1997, vol. 19, pp. 677-695.

[16] The datasheet of the spectrasymbol's flex sensor product.

[17] M. Petriska, D. Považanec, P. Fuchs, "Speech control for car using the TMS320C6701 DSP", $10^{\text {th }}$ International Scientific Conference, Bratislava, 2000, pp. 97-100.
[18] G. Bertini and M. Magrini, “A Prototype Lab Box with DSK'C6711/13 for Rapid DSP Algorithm Development", White Paper, Institute of Science and Technology of Information (ISTI-CNR) National Research Council Pisa, October 2005, Italy.

[19] F. Beritelli, S. Casale, and A. Cavallaro, "A Robust Voice Activity Detector for Wireless Communications Using Soft Computing", IEEE Journal on Selected Areas in Communications (JSAC), Special issue on Signal Processing for Wireless Communications, 1998, vol. 16, no. 9.

[20] R.I. Mabo, and W.R. Donadson,"Zero Crossing Measurements for Phonetic Recognition of Speech", IEEEASSP, vol. 24, no. 1.

[21] R.D. Schraft, C. Schaeffer, and T. May, "Care-O-bot: The Concept of a System for Assisting Elderly or Disabled Persons in Home Environments", In Proceedings of the 24th Annual Conference IECON98, 1998, vol. 4, pp. 2476-2481.

[22] J. Huang, N. Ohnishi, and N. Sugie, "Sound Localization in Reverberant Environment Based on the Model of the Precedence Effect", IEEE Transaction on Instrumentation and Measurement, 1997, vol. 46, pp. 842-846.

[23] E. Shlomot, V. Cuperman, and A. Gersho, "Hybrid Coding of Speech at 4 Kbps". In Proceeding of IEEE Workshop on Speech Coding for Telecommunication, Pocono Manor, PA, USA, September 1997, pp. $37-38$.

[24] T. Wang, and V. Cuperman, "Robust Voicing Estimation with Dynamic Time Warping". In Proceeding of IEEE International conference on Acoustic, Speech and Signal Processing, Seattle, WA, USA, May 1998, pp. 533-536.

[25] A. Rao, K. Rose and A. Gersho, "Deterministically Annealed Design of Speech Recognizers and Its Performance on Isolated Letters", Proceedings of the 1998 IEEE International Conference on Acoustics, Speech and Signal Processing, Seattle, WA, USA, May 1998, pp. 461-464.

[26] E.C. Ifeachor, and B.W. Jervis, Digital Signal Processing: A Practical Approach, second edition, Pearson Education, 2002, pp. 352-356.

[27] N.A. Weiss, Introductory Statistics, seventh edition, Pearson Education, 2005, pp. 724-727. 